\title{
Aerial target recognition based on Zernike moments of information fusion FU Tai ${ }^{1}$, SUN Xiangyi ${ }^{1}$, FU Dan ${ }^{2}$

\author{
College of Aerospace and Material, National University of Defense Technology, 410073, Changsha,
} China ${ }^{1}$
}

The Second Artillery Force of the Chinese People Liberation Army Model Office at the First Academy of China Aerospace Science and Technology Corporation, Beijing 100076 China $^{2}$

Keywords: OpenGL; Pseudo-Zernike; Pose Estimation;.

ABSTRACT :Based on OpenGL and Google library we established the target model library and the canny operator is used for edge detection. Combined with D-S evidence theory and Pseudo-Zernike moments, the air target recognition algorithm based on information fusion is proposed. Pseudo-Zernike moments are invariant to rotation, translation and scale invariance and easy to construct high order moment, compared with the Hu moment redundancy small, and not easy to affect by noise. The D-S theory of evidence solved the conflict absorption which make the result more reliable. The reliability and robustness of the method are verified by the simulation experiment of the target image in Gauss noise

\section{INTRODUCTION}

Object recognition is one of the basic tasks of machine vision application. The goal is to find a description of the effective information that can be used to distinguish different objects. As the object to be recognized is known in advance, the geometric features of the target object can be directly applied to the recognition task.

According to the different matching objects, the shape matching of irregular objects can be divided into the matching method based on region or the contour. Contour shape matching [11][12] is more commonly used in practice duo to two main reasons: one is that the contour features have less computation, which can meet the real-time requirements; two is that its geometric information can be used to identify with the target recognition information. In order to identify the irregular objects, the main task is to design an effective matching method based on the geometric constraints of (a small amount of) objects.

The theory of evidence [2] establishes a one-to-one correspondence between the propositions and sets, and deduces a set of mathematical theories about the reasoning of evidence, which is transformed into the problem of uncertainty of propositions.

The main work of this paper is:

1. Build the system model database of aircraft.

2. Design information fusion image matching algorithm, the actual captured images to match.

3 . Test the validity of the algorithm according to the results of the image matching.

\section{ALGORITHM}

a) Obtain the binary edge by Canny operator[3], where $n^{\in N}$ said the number of contour extraction in a single frame image.

b) Construct the aircraft shape sampling database offline. Denoting aircraft target profile model as $\boldsymbol{C}_{\boldsymbol{P B}}(a)$, where $a \in N^{+}$denotes the number of contours. In this paper, we render multi view images according to the three-dimensional model of the target, where the $5^{\circ}$ heading and pitch angle are the step size in the range of $(0,180)$.

c) With the observation distance of the air launch test much larger than the target's own dimension, the affine model of the linear transformation can be approximated by the nonlinear perspective 
transformation model. In this way, the shape will be described by the affine invariant boundary moment [5].

d) Offline compute the moment description $I_{i}^{P B}(a)$ of $\boldsymbol{C}_{\boldsymbol{P B}}(a)$, where $i \in \boldsymbol{N}^{+}$and $i \leq 3$. In this way, the shape model sample storage structure is denoted as follows:

\begin{tabular}{cccccc}
\hline$t$ & $v$ & $k$ & $l$ & $p_{i}$ & $I_{i}^{P B}(a)$ \\
\hline $\begin{array}{c}\text { Aircraft } \\
\text { model }\end{array}$ & $\begin{array}{c}\text { Observation } \\
\text { angle }\end{array}$ & $\begin{array}{c}\text { Numbers of } \\
\text { contour } \\
\text { sampling } \\
\text { points }\end{array}$ & $\begin{array}{c}\text { Predefined } \\
\text { number of } \\
\text { points }\end{array}$ & $\begin{array}{c}\text { Contour } \\
\text { sampling } \\
\text { points }\end{array}$ & $\begin{array}{c}\text { Affine } \\
\text { invariant } \\
\text { moments }\end{array}$ \\
\hline
\end{tabular}

Online calculate the moment description $I_{i}^{P R}(b)$ of $\boldsymbol{C}_{\boldsymbol{P R}}(b)$. Due to the possibility that PZM takes a non-positive boundary, the similarity between $\boldsymbol{C}_{\boldsymbol{P B}}(a)$ and $\boldsymbol{C}_{\boldsymbol{P R}}(b)$ was calculated with the modified chi-square distance $d(a, b)$ in which $\varepsilon$ is to deal with a possible case in which a denominator in formula becomes zero .

$$
d(a, b)=\left|\frac{1}{2} \sum_{i=1}^{3} \frac{\left[I_{i}^{P B}(a)-I_{i}^{P R}(b)\right]^{2}}{I_{i}^{P B}(a)+I_{i}^{P R}(b)+\varepsilon}\right|
$$

The cross-validation [8] was calculated to train the similarity threshold $\delta$, while the $d(a, b)$ sets to infinity which is larger than $\delta$. In this way, the minimum $d(a, b)$ corresponds to the outer moment of the aircraft contour is located in the region by non-minimum suppression in a single frame image.

e) Calculate the basic belief assignment function, and then use the theory of evidence "absorption"[5] method to get the final fusion, the fusion of the basic belief assignment function, and then calculate the maximum valve_— the final fusion after matching the target.

\section{Model Library Establishment}

To obtain the 2D projection of target in 3D digital model, the virtual camera may remain stationary in this thesis; so that the 2D projection image can be achieved at various poses as we rotate the object. In this article, OpenGL (Open Graphics Library, Silicon Graphics Inc.), the application programming interface (API) is utilized to perform multi-angle 2D projection on the 3D model of aircraft carrier target, some of which is download from Google [10] as shown in Figure 1

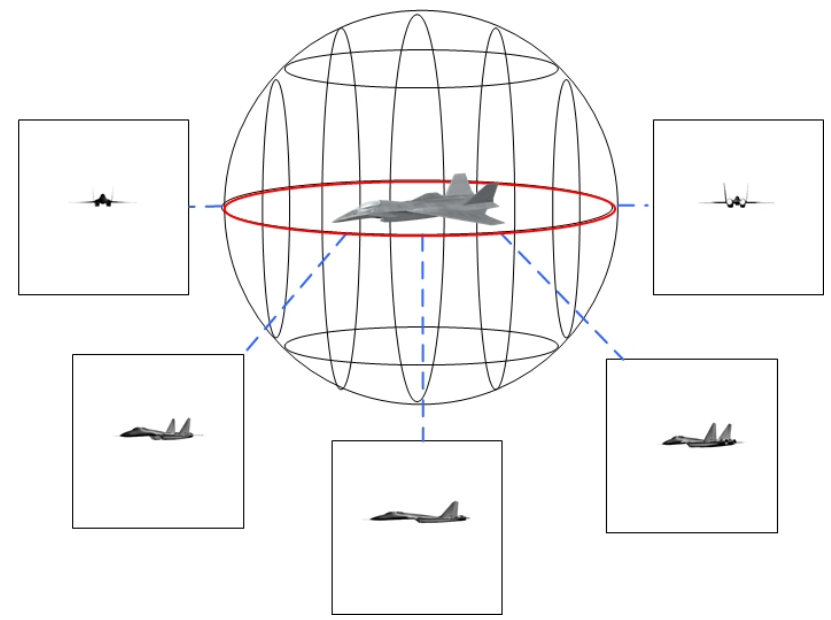

Figure 1. The 2D projective diagram of 3D model 


\section{Pseudo- Zernike Invariant Moment}

As an affine invariant moment, PZM [1]are derived from conventional Zernike moments[7] and shown to be more robust and less sensitive to image noise than the Zernike moments, which possess fine invariance towards translation, scaling, rotation and cropping. The PZM of order $n$ and repetition $l$ are defined as

$$
A_{n l}=\frac{n+1}{\pi} \int_{0}^{2 \pi} \int_{0}^{1}\left[V_{n l}(r \cos \theta, r \sin \theta)\right]^{*} f(r \cos \theta, r \sin \theta) r d r d \theta
$$

Where $n=0, \mathrm{~L} \infty$, and $l$ takes on positive and negative integer values subject to $|l| \leq n$. The image function can be reconstructed by expansion of the pseudo-Zernike coefficients on the unit disk as

$$
f(x, y)=\sum_{n=0}^{\infty} \sum_{l=-n}^{+n} A_{n l} V_{n l}(x, y)
$$

The task consists of the following steps:

a. The target image is segmented from the original image to find the minimum bounding rectangle of the region. Let length and width of the rectangle is $a$ and $b$ respectively, then shift the image center of gravity to the origin and embed into the unit circle with scaling the image by $2 / \sqrt{a^{2}+b^{2}}$ times.

b. Calculate the area of the target, the zero order moment

$$
m_{00}=\iint f(x, y) d x d y
$$

c. Calculate the Pseudo- Zernike moments in unit circle

$$
A_{n l}=\frac{n+1}{\pi} \sum_{x} \sum_{y} f(x, y) V_{n l}^{*}(\rho, \theta)
$$

d. Normalize Pseudo- Zernike moments, then take the modulus of Zernike moments $\left|A_{n m}\right|$ as the feature vector of image retrieval

$$
\left|A_{m l}\right|=\frac{\left|A_{m l}\right|}{m_{00}}
$$

\section{D-S Evidence Theory}

a) Frame of Discernment

Suppose the complete set of all possible answers that may be recognized is denoted by $\Theta=\left\{\theta_{1}, \mathrm{~L}, \theta_{N}\right\}$, in which the relationship between all of the two elements in the set is mutually exclusive.

b) Basic Trust Probability Assignment

$A$ and $m$ are standard for an any subset in the trust frame and the basic trust probability assignment, which satisfy that $m(\varnothing)=0$ and $\sum m(A)=1, A \subset \Theta$.

c) Combination Rules

Supposing that $m_{1}$ and $m_{2}$ denote the basic trust probability assignment corresponding to the evidence E1 and E2 in the frame of discernment separately, while their focal element are $A_{i}$ and $B_{j}$. Supposing $L$ is the threshold and the fusion rule [6] denotes by:

$$
\begin{aligned}
m(A)= & \sum_{A_{i} \cap B_{j}=A} m_{1}\left(A_{i}\right) m_{2}\left(B_{j}\right)+\sum_{\substack{A \cap A_{i}=\varnothing \\
m_{1}(A)>m_{2}\left(A_{i}\right)+L}} m_{1}(A) m_{2}\left(A_{i}\right)+\sum_{\substack{A \cap B_{j}=\varnothing \\
m_{1}(A)<m_{2}\left(B_{j}\right)-L}} m_{1}(A) m_{2}\left(B_{j}\right) \\
& +\sum_{\substack{A \cap A_{i}=\varnothing \\
\mid m_{1}(A)-m_{2}\left(A_{i}\right) \leq \leq L}} \frac{m_{1}(A) m_{2}\left(A_{i}\right)}{2}+\sum_{\substack{A \cap B_{j}=\varnothing \\
\left|m_{1}(A)-m_{2}\left(B_{j}\right)\right| \leq L}} \frac{m_{1}(A) m_{2}\left(B_{j}\right)}{2}
\end{aligned}
$$


The smaller the Euclidean distance of a certain type of object, the more closer to the trust assigned to the proposition, which is the overall trend that distance functions to the mapping of the basic trust distribution function when $R \rightarrow[0,1]$. Assignment equals to 1 when the distance is 0 , otherwise the assignment of this kind of proposition is more close to that of the other. In this way, where $\alpha$ is the noise factor the basic trust probability assignment is:

$$
m_{i}\left(F_{j}\right)=(1-\alpha) \exp \left(-d_{i j}\right)
$$

\section{EXPERIMENT}

The performance of the algorithm is demonstrated by the method of shape retrieval, feature location, and other interpretation methods.

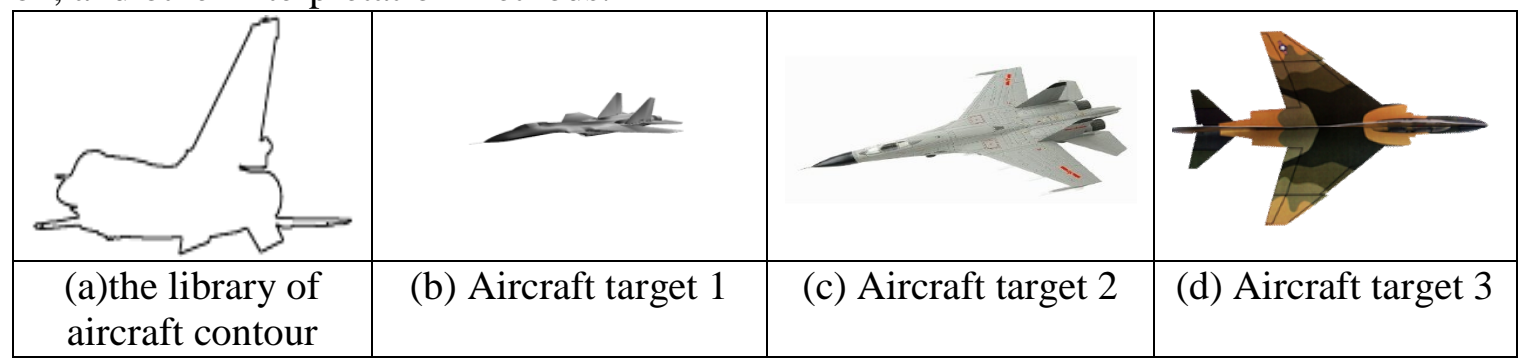

Figure 2. Aircraft sample library and target to be retrieved

White Gaussian noise is applied on the target contour which is retrieved on the sample databases corresponding to the Figure 2(b), (c) and (d) at the level of $(\mu, \sigma)$. The calculation of PZM of the image with noise and of the model image is as follows in

. The measurement results of the shape similarity between the image with noise and the model image demonstrated that the affine invariant moments are well described to express the similarity between shapes.

Table 1 Affine invariant moments of model images and with noisy images

\begin{tabular}{|c|c|c|c|c|c|c|c|c|}
\hline Target number & $\operatorname{noise}(\mu, \sigma)$ & $\overline{I_{1}}$ & $I_{2}$ & $I_{3}$ & $\begin{array}{l}\text { Model } \\
\text { number }\end{array}$ & $I_{1}$ & $I_{2}$ & $I_{3}$ \\
\hline 1 & $(0.0,0.0)$ & $5.43 \times 10^{-2}$ & $-1.73 \times 10^{-5}$ & $-1.24 \times 10^{-3}$ & $(1,3)$ & $8.17 \times 10^{-2}$ & $-5.41 \times 10^{-5}$ & $3.08 \times 10^{-3}$ \\
\hline 1 & $(0.0,0.5)$ & $5.92 \times 10^{-2}$ & $-2.12 \times 10^{-5}$ & $-1.45 \times 10^{-3}$ & $(1,3)$ & $8.17 \times 10^{-2}$ & $-5.41 \times 10^{-5}$ & $3.08 \times 10^{-3}$ \\
\hline 1 & $(0.0,1.0)$ & $6.67 \times 10^{-2}$ & $-3.17 \times 10^{-5}$ & $-1.75 \times 10^{-3}$ & $(1,4)$ & $1.72 \times 10^{-1}$ & $2.65 \times 10^{-3}$ & $2.29 \times 10^{-2}$ \\
\hline 2 & $(0.0,0.0)$ & $1.80 \times 10^{-1}$ & $-1.92 \times 10^{-5}$ & $2.44 \times 10^{-3}$ & $(1,3)$ & $8.17 \times 10^{-2}$ & $-5.41 \times 10^{-5}$ & $3.08 \times 10^{-3}$ \\
\hline 2 & $(0.0,0.5)$ & $1.91 \times 10^{-1}$ & $-2.09 \times 10^{-5}$ & $2.60 \times 10^{-3}$ & $(1,2)$ & $8.43 \times 10^{-2}$ & $-4.26 \times 10^{-6}$ & $1.19 \times 10^{-3}$ \\
\hline 2 & $(0.0,1.0)$ & $2.03 \times 10^{-1}$ & $-2.15 \times 10^{-5}$ & $2.69 \times 10^{-3}$ & $(1,6)$ & $9.59 \times 10^{-2}$ & $1.92 \times 10^{-4}$ & $-4.40 \times 10^{-3}$ \\
\hline
\end{tabular}

Table 2 Shape similarity measurement

\begin{tabular}{ccc}
\hline $\begin{array}{c}\text { Target } \\
\text { number }\end{array}$ & $\begin{array}{c}\text { Model } \\
\text { number }\end{array}$ & similarity \\
\hline 1 & $(1,3)$ & $2.36 \times 10^{-3}$ \\
1 & $(1,3)$ & $1.50 \times 10^{-3}$ \\
1 & $(1,4)$ & $1.28 \times 10^{-2}$ \\
2 & $(1,3)$ & $1.84 \times 10^{-2}$ \\
2 & $(1,2)$ & $2.04 \times 10^{-2}$ \\
2 & $(1,6)$ & $1.89 \times 10^{-2}$ \\
\hline
\end{tabular}

The basic confidence function is calculated by (7), where $\mathrm{M}$ represents the model of the aircraft that 1 stands for J20, 2 represents the su27 and 3 names the MiG 29. The result is as follows:

$\mathrm{m} 1 \_1,=0.9755, \mathrm{~m} 1 \_2,=0.0082, \mathrm{~m} 1 \_3,=0.0164$

$\mathrm{m} 2 \_1,=0.2350, \mathrm{~m} 2 \_2,=0.4940, \mathrm{~m} 2 \_3,=0.2710$ 
m3_1, $=0.2572, \mathrm{~m} 3 \_2,=0.4824, \mathrm{~m} 3 \_3,=0.2604$

Calculating the fused Basic trust allocation function and we can get that $\mathrm{m} 1=0.9860, \mathrm{~m} 2$ $=0.0091, \mathrm{~m} 3=0.0049$. Obviously the $\mathrm{m} 1$ is maximum, which demonstrate that the matching image of the aircraft model is $\mathbf{J} 20$.

\section{ACKNOWLEDGMENTS}

This work is supported by the National Key Scientific Instrument and Equipment Development Project Grant No. 2013YQ140517

\section{REFERENCES}

[1] Haddadnia, J.; Ahmadi, M.; Faez, K. (2003). "An efficient feature extraction method with pseudozernike moment in rbf neural network-based human face recognition system". EURASIP Journal on Applied Signal Processing. 2003 (9): 890-901. doi:10.1155/S1110865703305128.

[2] LAN Jinhui, MA Baohua, LAN Tian,ZHOU Zhaoying.D-S evidence reasoning and its data fusion application in target recognition[J]. Journal of Tsinghua University(Science and Technology), 2001, 41(2):53-55(Chinese)

[3] Deriche R. Using Canny's criteria to derive a recursively implemented optimal edge detector[J]. International Journal of Computer Vision, 1987, 1(2):167-187.

[4] Suzuki S. Topological structural analysis of digitized binary images by border following [J]. Computer vision, graphics, and image processing, 1985, 30 (1): 32-46.

[5] Flusser J, Suk T. Pattern recognition by affine moment invariants [J]. Pattern Recognition, 1993, 26 (1): 167-174.

[6] CHENG Yong-mei, PAN Quan, ZHANG Hong-cai, Wang Gang. Information fusion image recognition algorithum and applications in threee dimension airplane iamge recognition.[J]. Acta Aeronautica ET Astronautica Sinica, 2004, 25(2):176-179.(Chinese)

[7] Khotanzad A, Hong Y H. Invariant Image Recognition by Zernike Moments[J]. IEEE Transactions on Pattern Analysis \& Machine Intelligence, 1990, 12(5):489-497.

[8] Khotanzad A, Hong Y H. Rotation invariant image recognition using features selected via a systematic method[J]. Pattern Recognition, 1990,23 (10) : 1089 1101.

[9] Richard O D, Peter E H, David G S. Pattern classification [J]. A Wiley-Interscience Publication, 2001, 373378

[10] Google. Trimble 3D warehouse [EB/OL]. http://sketchup.google.com/3dwarehouse/, 2013-05$20 / 201$

[11] HU M K. Visual Pattern Recognition by Moment Invariants[J] .IEEE Trans on Information Theory, 1962, (8): 179 187

[12] M R Teague. Image analysis via the general theory of moments[J].Journal of the Optical Society of America,1980,70(08):920 930 